\title{
EFECTO DE LA SALINIDAD DEL SUELO SOBRE LA GERMINACIÓN DE SEMILLAS DE MAÍZ DE DIFERENTES PESOS EN EL ORIENTE VENEZOLANO
}

\section{EFFECT OF SOIL SALINTY ON SEED GERMINATION OF DIFFERENT WEIGHTS IN CORN IN EASTERN VENEZUELA}

\author{
José A. Laynez-Garsaball'1, Jesús Rafael Méndez¹ y Juliana Mayz-Figueroa \\ Recibido para evaluación: Agosto 15 de 2007 - Aceptado para publicación: Octubre 31 de 2007
}

\section{RESUMEN}

\begin{abstract}
El objetivo fue evaluar el efecto de diferentes niveles de salinidad del suelo sobre la germinación de semillas de diferentes pesos de dos cultivares de maíz en el Oriente de Venezuela. Se emplearon dos suelos salinos $(9.11$ y $15.19 \mathrm{dS} \mathrm{m}^{-1}$ de conductividad eléctrica (CE) determinada de un extracto relación 1:1. El testigo lo constituyó un suelo con CE de $0.13 \mathrm{dS} \mathrm{m}^{-1}$. La siembra se efectuó en bandejas de aluminio, empleando $10 \mathrm{~kg}$ de suelo por bandeja y 50 semillas. El diseño estadístico fue el de parcelas divididas con cuatro repeticiones, estando constituida la parcela principal por los potenciales osmóticos de los tres suelos $(-0.005,-0.328$ y $-0.547 \mathrm{MPa}$, correspondientes

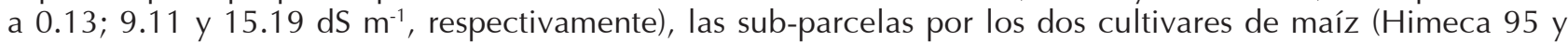
Pioneer 361) y las sub-sub-parcelas por tres pesos de semillas $(<0.32 \mathrm{~g}, 0.32-0.36$ g y >0.36 g). Se evaluó: porcentaje de germinación (\%), índice de la velocidad de germinación y número medio de días a total germinación. Un incremento en los niveles de salinidad causó una reducción de la germinación de las semillas y retardaron el proceso de germinación. Los potenciales osmóticos de los suelos salinos evaluados (-0.328 y -0.547 MPa) no diferenciaron la tolerancia a tal estrés en los dos cultivares de maíz en el proceso de germinación. El peso de la semilla no influenció el proceso de germinación en ninguno de los suelos.
\end{abstract}

Palabras Clave: Zea mays, masa de semillas, estrés salino, caracteres germinativos.

\begin{abstract}
The objective was to evaluate the effect of three seed sizes of two corn cultivars on the germination of seeds sowed in two saline soils (9.11 and $15.19 \mathrm{dS} \mathrm{m}^{-1}$ ) in Eastern Venezuela. The control was a soil with an EC of 0.13

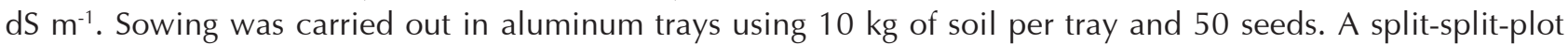
design was used with four replications, the main plots were the osmotic potentials (0.005 - 0.328 and - 0.547 $\mathrm{MPa}$, corresponding to $0.13,9.11$ y $15.19 \mathrm{dS} \mathrm{m}{ }^{-1}$, respectively), sub-plots were the two corn cultivars (Himeca 95 and Pioneer 361$)$ and sub-sub-plots were three seed weights $(<0.32 \mathrm{~g}, 0.32-0.36 \mathrm{~g}$ and $>0.36 \mathrm{~g})$. Germination percentage, germination speed index and mean number of days to final germination were recorded. The increased salinity level caused a decreasing in seed germination and delays in the germination process. Osmotic potentials of saline soil evaluated had no influence on salinity tolerance by the two corn cultivars in the germination stage. Seed weight had no effects on the germination process under the three evaluated soils.
\end{abstract}

Key words: Zea mays, seed mass, salt stress, germination traits.

Escuela de Ingeniería Agronómica, Núcleo Monagas, Universidad de Oriente, Avenida Universidad, Campus los Guaritos, Maturin 6201. Email: jalaynezg@yahoo.es; jmendezn@cantv.net; julianamays@cantv.net 


\section{INTRODUCCIÓN}

Las plantas están sometidas frecuentemente a situaciones desfavorables para su desarrollo y funcionamiento óptimo ocasionadas por alteraciones en el medio ambiente (AzconBieto y Talon, 1993). La salinización es un proceso de enriquecimiento del suelo con sales más solubles que el sulfato de calcio, por lo general se trata de cloruros y sulfatos de sodio y de magnesio. Esto provoca valores muy altos de la presión osmótica en el agua del suelo, con evidentes repercusiones sobre la vegetación (interfiere en el crecimiento de la mayoría de los cultivos y otras plantas no especializadas) (Porta et al., 1999).

Entre los estudios más detallados sobre la salinidad en Venezuela, Chirinos et al. (1991) detectaron problemas de salinidad en suelos de Cagua, Crespo, San Francisco de Asís, Santa Cruz y Villa de Cura en el estado Aragua; Guacara en el estado Carabobo; Yaritagua en e estado Yaracuy y Santa Rosalía en el estado Portuguesa, Venezuela. Mata y Pla (1992), basándose en evidencias y estudios previos, ubicaron, reconocieron a nivel de campo, muestrearon y analizaron suelos y aguas de riego en cuatro zonas con problemas actuales de afectación por sales, ubicadas en la Cuenca del Lago de Maracaibo, con el fin de realizar una caracterización con fines de diagnóstico del referido problema. Los resultados indicaron que las aguas de riego usadas en dichos sectores poseen una baja concentración de sales, indicando su evaluación que sólo causarían problemas de salinidad y/o sodicidad en condiciones de suelos con drenaje muy restringido. Las aguas de los ríos Limón y Motatan mostraron una mayor potencialidad de producir problemas de sodicidad. La caracterización de suelos indica que en el sector Río Limón predominan los suelos salinos, con influencia de aportes extras de cloruro de calcio. En el sector Río Palmar se detectaron suelos salinos y salino-sódicos con influencia de aportes extras de sulfato de sodio. En el sector El Cenizo predominan los suelos salino-sódicos con influencia de aportes extras de sulfato de sodio; en el sector Costa Oriental predominan los suelos salino-ácidos a diferentes niveles con influencia de sedimentos lacustrinos salobres.

Las pruebas de calidad de semillas buscan: predecir la vida de almacenamiento de un lote de semillas o su calidad después de un período especificado; predecir la emergencia en campo después de la siembra, y predecir el subsiguiente vigor de las plántulas y el rendimiento final del cultivo (Ellis y Roberts, 1980). La técnica de envejecimiento acelerado es la más usada para el logro de los objetivos anteriormente citados, aunque se le crítica por no estar estandarizada lo que ocasiona que pequeñas diferencias en el tratamiento de envejecimiento usado den lugar a variaciones en el vigor (Musgrove et al., 1980). Una alternativa sencilla a esta técnica para la identificación de semilla de buena calidad y al mismo tiempo para el estudio del efecto del estrés por salinidad pudiera ser el empleo de compuestos o productos comerciales para simular bajo condiciones de laboratorio el estrés salino: sulfato de sodio y cloruro de sodio (Martínez, 1999; Wong, 2002; Méndez et al., 2002). Su principio está en la premisa de que una semilla tenga capacidad para germinar y producir una plántula bajo condiciones de estrés salino es indicativo de un potencial genético para la tolerancia a la sal, al menos en esta etapa del ciclo de vida (Bernstein y Ayers, 1953; Pearson et al., 1966).

El cultivo de maíz se encuentra ampliamente distribuido en toda Venezuela, incluidos los suelos salinos. En consecuencia, es conveniente realizar investigación que permita identificar genotipos de maíz tolerantes $y / o$ resistentes a tales condiciones 
de estrés. Por otro lado, investigaciones sobre el efecto del estrés por salinidad, en genotipos de garbanzo (Soltani et al., 2002), indican variaciones en la germinación y en el crecimiento de las plántulas dentro de un mismo genotipo atribuidas a la influencia del tamaño de la semilla en la tolerancia a estas condiciones. Esto sugiere la posible selección de un determinado tamaño de semilla de un cultivar con objeto de obtener mayor tolerancia al estrés durante la germinación. Este experimento fue planificado para evaluar el efecto del potencial osmótico de suelos salinos sobre la germinación a partir de diferentes tamaños de semilla de dos cultivares de maíz, en condiciones de umbráculo.

\section{MATERIALES Y MÉTODOS}

\section{Ubicación}

La presente investigación se llevo a cabo en el umbráculo del Postgrado en Agricultura Tropical de la Universidad de Oriente, Estado Monagas, Venezuela. Fue estudiado el efecto del potencial osmótico de dos suelos salinos sobre el crecimiento de plántulas de maíz.

\section{Procedencia de los suelos}

Se usaron suelos no esterilizados (previamente secados al aire y cernidos a través de un tamiz de malla de $3 \mathrm{~mm}$ ), obtenidos de una franja de suelo entre los 5 y $20 \mathrm{~cm}$ de profundidad. Se emplearon dos suelos salinos (9.11 y 15.19 dS $\mathrm{m}^{-1}$, CE determinada de un extracto obtenido en una relación 1:1) provenientes de las cercanías de la Laguna de Chacopata, Municipio Cruz Salmerón Acosta, Estadodo Sucre. El testigo lo constituyó un suelo con CE de 0.13 dS $\mathrm{m}^{-1}$, proveniente de la Estación Biológica de Sabana de la U.D.O., Jusepín, estado Monagas. A los tres suelos se les realizó una estimación aproximada del potencial osmótico a partir de la conductividad eléctrica mediante la relación $y_{\text {os }}=-a C E$, donde la constante a para el cloruro de sodio es igual a 36 (Porta et al., 1999), es decir, los valores de potencial osmótico para los suelos con 0.13; 9 y $15 \mathrm{dS} \mathrm{m}^{-1}$ fueron -0.005; -0.328 y -0.547 , respectivamente.

\section{Características de los suelos}

En la tabla 1, se aprecian las características físicas y químicas de cada suelo, determinadas a través del análisis respectivo en el Laboratorio de Servicios Agroambientales (LABSEA) de la Universidad de Oriente. El suelo usado para la comparación con los suelos salinos presentó un potencial osmótico de -0.005 MPa, textura franco arenosa, bajo contenido de $\mathrm{P}$ y de cationes, (con excepción del calcio con contenido medio), bajo en M.O., y pH ácido. El suelo salino con potencial osmótico de -0.328 MPa presento una textura franco arenosa, mientras que la textura del suelo salino con potencial osmótico de 0.547 MPa fue arenosa, ambos suelos con un bajo contenido de $\mathrm{P}$, alto contenido de calcio y de magnesio, bajo de potasio y de M.O., y pH ligeramente alcalino.

\section{Cultivares, cribaje de la semilla}

Se utilizó semilla certificada de los cultivares comerciales de maíz: Himeca 95 y Pioneer 361 , con un contenido de humedad promedio de $12 \%$, tratadas con Vitavax $200 \mathrm{~F}^{\circledR}$ (carboxin $17 \%$ + thiram 17\%), para prevenir el crecimiento de hongos durante la germinación. Dos kilogramos de semillas de cada cultivar fueron sometidos a un proceso de selección por el que las semillas se separaron en base al peso individual, en tres intervalos de peso, en lotes de 300 semillas: $<0.32$ g, $0.32-0.36$ g y $>0.36$ g.

\section{Siembra y diseño experimental}

El presente trabajo se realizó en el umbráculo del Postgrado en Agricultura Tropical de la Universidad de Oriente (UDO), Estado Monagas, Venezuela. Se emplearon bandejas de aluminio $(41 \mathrm{~cm}$ largo, $26.5 \mathrm{~cm}$ ancho y $10 \mathrm{~cm}$ alto), desinfectadas con hipoclorito de sodio $(5.25 \%)$ y posteriormente lavadas con abundante agua para eliminar el exceso de 
Tabla 1. Características físicas y químicas de los suelos empleados en el ensayo de germinación de semillas a partir de tres tamaños de semilla de dos cultivares de maíz (Zea mays L.), bajo tres condiciones de suelo salino, en Monagas, Venezuela. +

Fertilidad

\begin{tabular}{|c|c|c|c|c|}
\hline Características & Unidad & Suelo 1: Jusepín & Suelo 2: Chacopata & Suelo 3: Chacopata \\
\hline $\mathrm{pH}$ & & 6.10 & 7.70 & 7.70 \\
\hline $\mathrm{P}$ & $\mathrm{Mg} \mathrm{Kg}^{-1}$ & 9.82 & 8.80 & 7.76 \\
\hline $\mathrm{Ca}^{++}$ & $\mathrm{cmol} \mathrm{Kg}^{-1}$ & 0.67 & 4.59 & 3.02 \\
\hline $\mathrm{Mg}^{++}$ & $\mathrm{cmol} \mathrm{Kg}^{-1}$ & 0.17 & 1.98 & 1.97 \\
\hline $\mathrm{K}^{+}$ & $\mathrm{cmol} \mathrm{Kg}^{-1}$ & 0.01 & 0.06 & 0.04 \\
\hline $\mathrm{Na}^{+}$ & $\mathrm{cmol} \mathrm{Kg}^{-1}$ & Traza & 8.78 & 11.17 \\
\hline $\mathrm{Al}^{+++}$ & $\mathrm{cmol} \mathrm{Kg}^{-1}$ & Traza & Traza & Traza \\
\hline $\mathrm{H}^{+}$ & $\mathrm{cmol} \mathrm{Kg}^{-1}$ & 0.09 & 0.17 & 0.17 \\
\hline$(\mathrm{H}+\mathrm{Al})$ & $\mathrm{cmol} \mathrm{Kg}^{-1}$ & 0.09 & 0.17 & 0.17 \\
\hline CICE & $\mathrm{cmol} \mathrm{Kg}^{-1}$ & 0.94 & 15.60 & 16.40 \\
\hline$\%$ SAT AL & $\%$ & - & - & - \\
\hline M.O. & $\%$ & 0.94 & 0.86 & 0.37 \\
\hline \multicolumn{5}{|c|}{ Textura } \\
\hline Arcilla & $\%$ & 9.20 & 3.20 & 3.20 \\
\hline Clase & & Areno franco $(\mathrm{aF})$ & Areno franco $(\mathrm{aF})$ & Arenoso (a) \\
\hline \multicolumn{5}{|c|}{ Salinidad } \\
\hline $\mathrm{pH}$ & & 5.000 & 7.70 & 7.70 \\
\hline CE & $\left(\mathrm{mmhos} \mathrm{cm}^{-1}\right)$ & - & - & - \\
\hline Sulfatos & $\left(\right.$ me l-1) $\left.^{-1}\right)$ & 0.110 & 2.35 & 3.75 \\
\hline Carbonatos & $\left(\mathrm{me} \mathrm{l}^{-1}\right)$ & Traza & Traza & Traza \\
\hline Bicarbonatos & $\left(\mathrm{me} \mathrm{l}^{-1}\right)$ & 0.190 & 0.54 & 0.32 \\
\hline Cloruros & $\left(\right.$ me l-1 $\left.^{-1}\right)$ & 0.370 & 27.69 & 30.63 \\
\hline Calcio & $\left(\mathrm{me} \mathrm{I}^{-1}\right)$ & 0.426 & 3.40 & 3.94 \\
\hline Magnesio & $\left(\mathrm{me} \mathrm{l}^{-1}\right)$ & 0.215 & 5.38 & 5.04 \\
\hline Potasio & $\left(\mathrm{me} \mathrm{I}^{-1}\right)$ & 0.014 & 0.45 & 0.42 \\
\hline Sodio & $\left(\mathrm{me} \mathrm{l}^{-1}\right)$ & 0.004 & 25.86 & 27.21 \\
\hline RAS & & 0.010 & 12.31 & 12.83 \\
\hline
\end{tabular}

+ Laboratorio de Servicios Agroambientales (LABSAE) de la Universidad de Oriente

desinfectante. Cada bandeja con $10 \mathrm{~kg}$ de suelo y 50 semillas arregladas en lotes de 25 de acuerdo al tratamiento correspondiente (repetición, suelo, cultivar, tamaño de la semilla), distribuidas en 5 hileras de 5 semillas c/u (distancia entre plantas $3,70 \mathrm{~cm}$ y entre semillas $4,00 \mathrm{~cm}$ ) y a $3 \mathrm{~cm}$ de profundidad.
Se efectuaron riegos diarios de $250 \mathrm{ml}$ de agua en cada bandeja.

Se utilizó el diseño estadístico de parcelas divididas con 4 repeticiones, donde la parcela principal la conformaron los potenciales osmóticos de los tres suelos $(-0.005,-0.328$ 
y -0.547 MPa, correspondientes a $0.13 ; 9.11$ y $15.19 \mathrm{dS} \mathrm{m}^{-1}$, respectivamente), las subparcelas, los dos cultivares de maíz (Himeca 95 y Pioneer 361), y las sub-sub-parcelas, los tres tamaños de las semillas $(<0.32 \mathrm{~g}, 0.32-$ 0.36 g y $>0.36$ g).

\section{Variables medidas y análisis estadístico}

Los parámetros con los que se evalúo las respuestas de los cultivares a los diferentes tratamientos fueron:

Porcentaje de germinación (\%): a los 4, 8, 12 y 16 días después de la siembra (dds)

Índice de velocidad de germinación (IVG): estimado a través de la fórmula:

IVG $\left.=\left(N_{1} \times 4+N_{2} \times 4+\ldots+N_{n} \times\right) / T_{n}\right)$ (Hartmann et al., 1993).

Número medio de días a germinación (NMDG): determinado por la fórmula:

$\mathrm{NMDG}=\left(\mathrm{N}_{1} \times \mathrm{T}_{1}+\mathrm{N}_{2} \times \mathrm{T}_{2}+\ldots+\mathrm{N}_{\mathrm{n}} \times \mathrm{T}_{\mathrm{n}}\right) /$ n) (Khan y Ungar, 1984).
$\mathrm{N}=$ número de semillas germinadas dentro de los intervalos de tiempo consecutivos.

$\mathrm{T}=$ tiempo transcurrido entre el inicio de la prueba y el fin del intervalo

$\mathrm{n}=$ número de semillas germinadas.

Los resultados de los parámetros evaluados fueron analizados mediante el análisis de varianza convencional. Los datos del porcentaje de germinación fueron transformados mediante la fórmula $\sqrt{(X+3 / 8) /(n+3 / 4)}$. Las diferencias entre las medias se establecieron a través de la Prueba de Rangos Múltiples de Duncan al 5\% de probabilidad.

\section{RESULTADOS Y DISCUSIÓN}

\section{Porcentaje de germinación}

Los análisis de varianza para el porcentaje de germinación de semillas a los 4, 8, 12 y 16 días después de la siembra (dds) (Tabla 2), únicamente señalan efecto significativo para el

Tabla 2. Análisis de varianza para el porcentaje de germinación de semillas a los 4, 8, 12 y 16 días después de la siembra (dds) de tres tamaños de semilla de dos cultivares de maíz (Zea mays L.), bajo tres condiciones de suelo salino. Datos transformados mediante

\begin{tabular}{|c|c|c|c|c|c|}
\hline \multirow[b]{2}{*}{ Fuente de variación } & \multirow[b]{2}{*}{ GL } & \multicolumn{4}{|c|}{ Cuadrados Medios (DDS) } \\
\hline & & 4 & 8 & 12 & 16 \\
\hline Repetición & 3 & $0.374 \mathrm{~ns}$ & $4.278 \mathrm{~ns}$ & $4.364 \mathrm{~ns}$ & $3.457 \mathrm{~ns}$ \\
\hline Potencial osmótico (P) & 2 & $4.788 *$ & $35.378 *$ & $10.554 *$ & $5.471 \mathrm{~ns}$ \\
\hline Error (a) & 6 & 0.185 & 1.803 & 1.140 & 1.320 \\
\hline Cultivar de maíz (C) & 1 & $0.229 \mathrm{~ns}$ & $3.952 \mathrm{~ns}$ & $4.608 \mathrm{~ns}$ & $5.708 \mathrm{~ns}$ \\
\hline$P \times C$ & 2 & $0.627 \mathrm{~ns}$ & $4.753 \mathrm{~ns}$ & $4.196 \mathrm{~ns}$ & $1.810 \mathrm{~ns}$ \\
\hline Error (b) & 9 & 0.386 & 2.501 & 1.870 & 1.879 \\
\hline Tamaño de semilla $(\mathrm{T})$ & 2 & $0.116 \mathrm{~ns}$ & $0.255 \mathrm{~ns}$ & $0.579 \mathrm{~ns}$ & $0.674 \mathrm{~ns}$ \\
\hline $\mathrm{P} \times \mathrm{T}$ & 4 & $0.663 \mathrm{~ns}$ & $1.172 \mathrm{~ns}$ & $1.396 \mathrm{~ns}$ & $1.479 \mathrm{~ns}$ \\
\hline $\mathrm{C} \times \mathrm{T}$ & 2 & $0.340 \mathrm{~ns}$ & $0.787 \mathrm{~ns}$ & $2.170 \mathrm{~ns}$ & $2.061 \mathrm{~ns}$ \\
\hline $\mathrm{P} \times \mathrm{C} \times \mathrm{T}$ & 4 & $0.204 \mathrm{~ns}$ & $0.929 \mathrm{~ns}$ & $0.661 \mathrm{~ns}$ & $0.596 \mathrm{~ns}$ \\
\hline Error (c) & 36 & 0.346 & 0.979 & 0.786 & 0.734 \\
\hline Total & 71 & & & & \\
\hline C. V. (a) (\%) & & 5.80 & 13.75 & 10.14 & 10.70 \\
\hline C. V. (b) (\%) & & 8.38 & 16.19 & 12.99 & 12.77 \\
\hline C. V. (c) $(\%)$ & & 7.93 & 10.13 & 8.42 & 7.98 \\
\hline
\end{tabular}

$\mathrm{GL}=$ Grados de Libertad $\quad *$ : Significativo $(\operatorname{Pr} £ 0,05) \quad$ ns: No significativo $(\operatorname{Pr} 0.05)$ 
Tabla 3. Promedios para los porcentajes de germinación a los 4, 8 y 12 días después de la siembra de tres tamaños de semilla de dos cultivares de maíz (Zea mays L.), bajo tres condiciones de suelo salino.

\begin{tabular}{|c|c|c|c|}
\hline \multirow[b]{2}{*}{ Potencial Osmótico (MPa) } & \multicolumn{3}{|c|}{ Porcentaje de germinación (dds) } \\
\hline & 4 & 8 & 12 \\
\hline$-0.005(0.13)+$ & $47.65 \mathrm{~A} \neq$ & $59.50 \mathrm{~A}$ & $61.67 \mathrm{~A}$ \\
\hline$-0.328(9.11)$ & $13.83 \mathrm{~B}$ & $31.33 \mathrm{~B}$ & $47.00 \mathrm{~B}$ \\
\hline$-0.547(15.19)$ & $9.99 \quad \mathrm{~B}$ & $24.33 \mathrm{~B}$ & $40.50 \mathrm{~B}$ \\
\hline
\end{tabular}

† Valores entre paréntesis denotan la conductividad eléctrica en dS/m

₹ Prueba de Rangos Múltiples de Duncan $(\operatorname{Pr} £$ 0,05). Letras diferentes indican promedios estadísticamente diferentes dentro de una misma columna.

factor potencial osmótico a los 4, 8 y 12 dds, en tanto que a los 16 dds no hubo diferencias significativas en ninguna de las fuentes de variación con un promedio general de 51.92\%. Los promedios para el porcentaje de germinación a los 4, 8 y 12 dds (Tabla 3), indican que la mayor germinación se presentó en el suelo utilizado como testigo ( $y_{\text {os }}=-0.005 \mathrm{MPa}$ ), seguida por los suelos con potenciales osmóticos de -0.328 y -0.547 MPa, estadísticamente iguales entre sí e inferiores al primero.

\section{Índice de la velocidad de germinación}

El análisis de varianza (Tabla 4) muestra diferencias significativas sólo para el factor potencial osmótico. En la tabla 5 se presenta la prueba de promedios, en la que puede observarse que el mayor índice de velocidad de germinación se presentó en el suelo empleado como testigo $\left(\mathrm{y}_{\text {os }}=-0.005 \mathrm{MPa}\right)$, superior estadísticamente a los suelos con potenciales osmóticos de -0.328 y $-0.547 \mathrm{MPa}$, ambos similares entre sí.

Tabla 4. Análisis de varianza para el índice de velocidad de germinación (IVG) y número medio de días a total germinación (NMDTG) de tres tamaños de semilla de dos cultivares de maíz (Zea mays L.), bajo tres condiciones de suelo salino.

\begin{tabular}{lccc}
\hline \multicolumn{1}{c}{ Fuente de variación } & GL & IVG & Cuadrados Medios \\
\hline Repetición & 3 & $41.898 \mathrm{~ns}$ & $1.351 \mathrm{~ns}$ \\
Potencial osmótico (P) & 2 & $169.070^{*}$ & $100.320^{*}$ \\
Error (a) & 6 & 16.270 & 1.768 \\
Cultivar de maíz (C) & 1 & $47.531 \mathrm{~ns}$ & $0.006 \mathrm{~ns}$ \\
P x C & 2 & $42.383 \mathrm{~ns}$ & $5.222 \mathrm{~ns}$ \\
Error (b) & 9 & 20.536 & 3.926 \\
Tamaño de semilla (T) & 2 & $5.694 \mathrm{~ns}$ & $2.434 \mathrm{~ns}$ \\
Px T & 4 & $17.280 \mathrm{~ns}$ & $0.627 \mathrm{~ns}$ \\
C x T & 2 & $16.461 \mathrm{~ns}$ & $0.408 \mathrm{~ns}$ \\
P x C x T & 4 & $8.432 \mathrm{~ns}$ & $1.118 \mathrm{~ns}$ \\
Error (c) & 36 & 8.289 & 2.180 \\
Total & 71 & & \\
C. V. (a) (\%) & & 13.340 & 7.420 \\
C. V. (b) (\%) & & 14.980 & 8.240 \\
C. V. (c) (\%) & & 9.520 &
\end{tabular}

$\mathrm{GL}=$ Grados de Libertad $\quad *$ : Significativo ( Pr£ 0.05) ns: No significativo ( $\operatorname{Pr}>0.05)$ 
Número medio de días para la germinación El análisis de varianza para el número medio de días para la germinación calculados en función de los porcentajes de germinación a los 4, 8, 12 y 16 dds (Tabla 4) señala significación estadística sólo para el factor potencial osmótico. La prueba de separación de medias (Tabla 5) muestra un mayor número medio de días para la germinación en el suelo con potencial osmótico de - $0.547 \mathrm{MPa}$, estadísticamente igual al suelo con potencial osmótico de $-0.328 \mathrm{MPa}$, y ambos superiores al suelo testigo ( $\mathrm{y}_{\text {os }}=-0.005 \mathrm{MPa}$ ).

Tabla 5. Promedios para el índice de la velocidad de germinación (IVG) y el número medio de días a total germinación (NMDTG) de tres tamaños de semilla de dos cultivares de maíz (Zea mays L.), bajo tres condiciones de suelo salino.

\begin{tabular}{ccc}
\hline Potencial Osmótico $(\mathbf{M P a})$ & IVG & NMDTG \\
\hline$-0.005(0.13)+$ & $12.16 \mathrm{~A} \neq$ & $5.8 \mathrm{~A}$ \\
$-0.328(9.11)$ & $8.40 \mathrm{~B}$ & $9.1 \mathrm{~B}$ \\
$-0.547(15.19)$ & $7.03 \mathrm{~B}$ & $9.6 \mathrm{~B}$ \\
\hline
\end{tabular}

† Valores entre paréntesis denotan la conductividad eléctrica en dS/m

‡ Prueba de Rangos Múltiples de Duncan ( Pr£ 0.05). Letras diferentes indican promedios estadísticamente diferentes dentro de una misma columna.

\section{DISCUSIÓN}

En el ensayo no se presentaron interacciones en los parámetros de germinación porcentaje de germinación, índice de velocidad de germinación y número medio de días para la germinación, observándose únicamente el efecto simple del factor potencial osmótico (nivel de salinidad). Esto significa, que las diferencias en los caracteres se debieron exclusivamente a la salinidad del suelo, es decir, los incrementos en los niveles de salinidad produjeron una disminución en la germinación de semillas, no existiendo influencia de un factor sobre otro. De acuerdo a Dodd y Donovan (1999), un incremento de la salinidad generalmente reduce la germinación, dos procesos regulan esta reducción: los efectos osmóticos debidos a una disminución del potencial de solutos del suelo, creando un estrés hídrico para la planta y los efectos iónicos debidos a la absorción y/o acumulación de iones por la semilla o las plántulas.

Las disminuciones de la germinación inducidas por la salinidad son usualmente debidas solamente a los efectos osmóticos (potencial de soluto del sustrato) para las halófitas, mientras que las glicofitas (como el maíz) son más probables a exhibir toxicidad iónica adicional. Sin embargo, Prisco y O'Leary (1970) indicaron que un alto contenido de sales en el suelo, especialmente cloruro de sodio, puede inhibir la germinación no solamente debido a la sequía fisiológica como también debido a una disminución del potencial hídrico sino también debido al aumento de la concentración de iones en el embrión, ocasionando un efecto tóxico. En este experimento no se determinó el proceso de imbibición de las semillas durante los tratamientos con salinidades diferentes, pero se sabe que una reducción en el porcentaje de germinación de las semillas en condiciones de déficit hídrico es atribuida a una menor difusión del agua a través del tegumento, el déficit hídrico ocasiona una prolongación de la fase estacionaria del proceso de imbibición por causa de la reducción de la actividad enzimática y consecuentemente, un menor desenvolvimiento meristemático y atraso en la profusión de la radícula (Falleri, 1994). Shonjani 
(2002) en un trabajo para determinar el efecto de diferentes niveles de salinidad $(\mathrm{NaCl})$ sobre la germinación de semillas de maíz cv. Pionner 3906 encontró que la imbibición de agua a partir de los tratamientos de $\mathrm{NaCl}(50,100$ y 200 mM) disminuyó debido al bajo potencial hídrico externo de la solución, concentraciones más altas de $\mathrm{NaCl}(200 \mathrm{mM})$ resultaron en menos imbibición de agua que las concentraciones más bajas de $\mathrm{NaCl}$, a un bajo potencial osmótico, el $\mathrm{NaCl}$ inhibió el proceso de imbibición y la caída en la tasa de absorción de agua, cuando las semillas se sumergieron en las soluciones de $\mathrm{NaCl}$ de concentración mayor es probablemente, causado por la disminución en el gradiente del potencial de agua entre las semillas y su medio circundante.

No se pudo diferenciar para resistencia a la salinidad entre los cultivares a nivel de germinación en los dos potenciales osmóticos evaluados (-0.328 y $-0.547 \mathrm{MPa})$ de los suelos salinos utilizados con conductividades eléctricas de 9.11 y 15.19 dS $\mathrm{m}^{-1}$, respectivamente. La explicación a la imposibilidad de discriminar a nivel de germinación entre cultivares para la tolerancia a la salinidad en estos suelos, pudiera estar en dos aspectos, en los suelos salinos utilizados existieron factores ligados al contenido de arcilla, materia orgánica y a los diferentes elementos en la solución del suelo que enmascararon en la etapa de germinación las posibles diferencias entre los cultivares, o bien, los potenciales osmóticos de los suelos de -0.328 y $-0.547 \mathrm{MPa}\left(9.11\right.$ y 15.19 dS $\mathrm{m}^{-1}$, respectivamente) no son adecuados para establecer diferencias entre los cultivares a nivel de este sustrato. Existió una disminución en la germinación por efecto del incremento de la salinidad (disminución del potencial osmótico) entre el suelo empleado para la comparación y los suelos salinos, aunque estos últimos no presentaron diferencias significativas entre sí. Porta et al., (1999), señalan que la presencia de sales en el suelo provoca un retardo en la nascencia, que con salinidades elevadas puede no tener lugar. Mientras que Daubenmire (1990), indicó que en condiciones salinas en la mayoría de las halófitas y las glicófitas la germinación es muy lenta y la supervivencia de las plántulas es muy difícil. Según Bansal et al. (1980), la germinación, en potenciales hídricos altamente negativos, especialmente al comienzo de la imbibición pudieran influenciar la absorción de agua por las semillas y este evento pudiera tornar no viable al proceso de germinación. Por otra parte, Larcher (1986) indicó que el exceso de los iones de sodio y cloro tiende a causar intumescencia protoplasmática, afectando la actividad enzimática, dando principalmente como resultado una producción inadecuada de la energía por disturbios en la cadena respiratoria.

La salinidad influencia significativamente la respuesta germinativa de la semilla, un exceso de sales solubles provoca una reducción del potencial hídrico del suelo, induciendo una menor capacidad de absorción de agua por las semillas. Esta reducción del potencial hídrico y de los efectos tóxicos de las sales interfiere inicialmente en el proceso de absorción de agua por las semillas influenciando la germinación (Cavalcante y Perez, 1995). Una reducción en el porcentaje de germinación y un atraso en el inicio del proceso germinativo con el aumento del estrés salino puede estar relacionado con una sequía fisiológica producida, pues cuando existe un aumento de la concentración de sales en el medio germinativo, hay una disminución del potencial osmótico y consecuentemente, una reducción del potencial hídrico (Fanti y Perez, 2004). Esta reducción puede afectar la cinética de absorción de agua por las semillas (efecto osmótico), como también elevar a niveles tóxicos las concentraciones de iones (efecto tóxico) (Cramer et al., 1986; Tobe et al., 2000). La inhibición de la movilización de las reservas puede ser atribuida a los 
efectos de las sales en la síntesis "de novo" y la actividad de enzimas responsables de la hidrólisis y translocación de los productos hidrolizados de los tejidos de reserva para el eje embrionario, afectando de este modo el proceso germinativo (Prisco et al., 1981). Moterle et al. (2006) indicaron que la disponibilidad de agua y el movimiento de agua para las semillas son muy importantes para la germinación y emergencia de plántulas y estos factores están influenciados por el potencial hídrico del suelo, la textura del suelo y la superficie de contacto del suelo con la semilla.

Otros investigadores, trabajando en condiciones de laboratorio con semillas de maíz tratadas con potenciales osmóticos creados con soluciones salinas han observado reducciones en la germinación producto del potencial osmótico y posiblemente por la toxicidad de las sales, así como diferencias entre cultivares. Moterle et al. (2006) indicaron que la reducción en el potencial osmótico redujo el comportamiento de la semillas de maíz de cotufa y hubo un comportamiento diferencial entre los cultivares de maíz de cotufa de la tolerancia al estrés salino. Mientras que da Silva et al., (2007) encontraron que la germinación y la tasa de germinación de semillas de cebada disminuyeron a medida que se incrementó el nivel de salinidad, reduciendo la viabilidad y vigor de las semillas debido a que la salinidad afectó la integridad de la membrana principalmente en el cultivar AF 98067 el cual mostró ser más susceptible al estrés salino. Méndez et al. (2002), evaluaron a nivel de laboratorio el efecto sobre tres cultivares de maíz (Cargill 633, Himeca 2003 y Pioneer 3031) de potenciales osmóticos $(0,-3,-6,-9$ y -12 Bares) creados con sulfato de sodio y observaron que el porcentaje de germinación se incrementó $9,45 \%$ a -3 bares, para luego disminuir con reducciones del potencial, la germinación se redujo 83,72 y 73,28 \% a -9 y -12 bares, respectivamente. Mientras que Martínez (1999), comparó el porcentaje de germinación entre los cultivares Cargill 717 y Cargill 633, y obtuvo un mayor porcentaje de germinación con Cargill 717 a potencial de agua de -0,03 MPa. En este experimento, las semillas se sembraron en suelos salinos hasta 15.19 dS m-1 y -0.547 Mpa. Según Murillo-Amador y Troyo-Diéguez (2000) en muchas especies estos valores son demasiados altos para la absorción de agua, la imbibición y germinación de las semillas, los resultados obtenidos a pesar de que el tratamiento control (-0.005 MPa), superó a las dos tratamientos salinos, la germinación de las semillas no fue inhibida y sólo disminuyó aproximadamente 23.79 y $34.22 \%$ para -0.328 y $-0.547 \mathrm{MPa}$, respectivamente a los 12 dds, mientras que a los 16 dds no hubo diferencia entre los tres suelos evaluados, estos datos sugieren que el maíz es un cultivo tolerante a la salinidad en la fase de germinación.

La disminución del porcentaje de germinación de las semillas de maíz, así como la disminución de la velocidad de germinación y el aumento del número medio de días para que ocurriera la germinación de todas las semillas (siendo estos dos últimos caracteres relacionados al vigor de la semilla) con incrementos en el nivel de salinidad ha sido reportada también en otra gramínea como el arroz. Rodrigues et al. (2002) indicaron que los tratamientos de salinidad del agua de riego con una conductividad eléctrica de $8.5 \mathrm{dS}$ $\mathrm{m}^{-1}$ deben haber reducido el potencial osmótico $\left(\varnothing_{\mathrm{o}}\right)$ de la solución del suelo notablemente, afectando el proceso de imbibición por las semillas de arroz, siendo este efecto más marcado al quinto día, atrasando la germinación y según Basnayake et al. (1994), la ocurrencia excesiva de sales solubles en el suelo causa una reducción en el potencial osmótico y como consecuencia, una reducción en el gradiente del potencial entre el suelo y la semilla, dificultando el proceso de imbibición y comprometiendo la germinación. Según Bruni y Leopold (1992), la reducción de la velocidad de la 
germinación y el incremento del número de días a germinación, probablemente, fue el resultado de la pérdida de turgencia protoplasmática, que provoca disturbios en la fisiología celular y daños en los sistemas de las biomembranas.

No se presentó influencia del tamaño de la semilla en la germinación por efecto del nivel de salinidad, ni del cultivar. Por lo que el tamaño de la semilla no parece ser un factor de importancia ligado al proceso de germinación bajo los niveles de sal (potenciales osmóticos salinos) con los que se trabajó en esta investigación. Esto coinciden con lo encontrado por Soltani et al. (2002), quienes estudiaron el efecto del tamaño de la semilla (grande, mediana y pequeña) y la salinidad (potencial osmótico de $0,-0.3,-0.6$ y -0.9 MPa) sobre la germinación de dos genotipos de garbanzo (Cicer arietinum L.).

Dantas et al. (2005) han indicado que aunque existe una gran cantidad de literatura describiendo los efectos de la salinidad en plantas adultas de fríjol, sin embargo, sus efectos durante la germinación de las semillas permanece aún inciertos, debido a este hecho más estudios con la germinación de semillas estresadas por salinidad son necesarios para una elucidación completa de su efecto sobre el desarrollo del fríjol. Esto mismo puede aplicarse al cultivo de maíz donde se han realizado numerosos ensayos evaluando la germinación de semillas y el crecimiento y desarrollo de plántulas y plantas, pero los mecanismos de la acción de las sales sobre estos caracteres son todavía contradictorios. También, es necesario realizar ensayos con suelos salinos en los que se evalúe una mayor cantidad de cultivares y/o niveles de salinidad (potenciales osmóticos) para tratar de establecer en cuales es posible discriminar para efectos de germinación.

\section{CONCLUSIONES}

- Los potenciales osmóticos de los suelos salinos evaluados (-0.328 y $-0.547 \mathrm{MPa}$, correspondientes a 9.11 y $15.19 \mathrm{dS} \mathrm{m}^{-1}$, respectivamente) no diferenciaron la tolerancia a tal estrés en los dos cultivares de maíz (Himeca 95 y Pioneer 361) durante la germinación.

- El incremento de la salinidad del suelo afectó negativamente tanto la cantidad de semillas germinadas como el tiempo para que ocurriera la germinación.

- El tamaño de la semilla no influenció el proceso de germinación en ninguna de los suelos salinos en estudio.

\section{AGRADECIMIENTO}

Al Consejo de Investigación de la Universidad de Oriente, Venezuela y al Postgrado en Agricultura Tropical del Núcleo Monagas de la Universidad de Oriente.

\section{BIBLIOGRAFÍA}

Azcon-Bieto, J. y Talon, M. 1993. Fisiología y Bioquímica Vegetal. Interamericana McGraw-Hill, Madrid, p537-539

\author{
Bansal, R.; Bhati, P. y Sem, D. 1980. \\ Differential specificity in water \\ inhibition of Indian arid zone. Biologia \\ Plantarum 22:327-331
}


Basnayake, J.; Cooper, M.; Ludlow, M. y Henkell, R. 1994. Combining ability variation for osmotic adjustment among a selected range of grain sorghum (Sorghum bicolor (L) Moench). Field Crops Research 38:147-155

Bernstein, L. y Ayers, A. 1953. Salt tolerance of five varieties of carrots. Proceedings of American Society for Horticultural Sciences 61:360-366

Bruni, F. y Leopold, A. 1992. Cytoplasmic glass formation in maize embryos. Seed Science Research 2(4):251-253

Cavalcante, A. y Perez, S. 1995. Efeitos dos estresses hídrico e salino sobre a germinação de sementes de Leucaena leucocephala (Lam.) de Witt. Pesquisa Agropecuária Brasileira 30(2):281-289

Cramer, G.; Läuchli, A. y Epstein, E. 1986. Effects of $\mathrm{NaCl}$ and $\mathrm{CaCl}_{2}$ on ion activities in complex nutrient solutions and root growth of cotton. Plant Physiology 81:792-797

Chirinos, A., De Brito, J. y De Rojas, E. 1971. Características de fertilidad de algunos suelos Venezolanos vistos a través de los resúmenes de análisis rutinarios. Agronomía Tropical 21(5):397-409

Dantas, B.; De Sá Ribeiro, L. y Aragão, C. 2005. Physiological response of cowpea seeds to salinity stress. Revista Brasileira de Sementes 27(1):144-148

Daubenmire, R. 1990. Ecología Vegetal. Limusa, México, D.F. p66

Dodd, G. y Donovan, L. 1999. Water potential and ionic effects on germination and seedling growth of two cold desert shrubs. American Journal of Botany 86(8):1146-1153
Ellis, R. y Roberts, E. 1980. Toward a national basis for testing seed quality. Seed Production, Hebblethwaite, p605-635

Falleri, E. 1994. Effect of water stress on germination in six provenances of Pinus pinaster Ait. Seed Science and Technology 22(3):591-599

Fanti, S. y Perez, S. 2004. Processo germinativo de sementes de paineira sob estresses hídrico e salino. Pesquisa Agropecuária Brasileira 39(9): 903-909

Hartmann, H.; Kester, E. y Davies, F. 1993. Plant Propagation, Principles and Practices. Prentice-Hall, New Delhi, p647

Khan, M. y Ungar, I. 1984. The Effect of salinity and temperature on the germination of polymorphic seeds and growth of Atriplex triangularis Willd. American Journal of Botany 71(4):481-489

Larcher, W. 1986. Ecofisiologia Vegetal. São Paulo, EPU, p319

Martínez A. 1999. Efecto de la temperatura y del contenido de agua del suelo en la germinación y crecimiento inicial en dos cultivares de maíz (Zea mays L.) con diferentes contenidos de humedad inicial en las semillas. Tesis M.Sc., Universidad de Oriente, Monagas

Mata R, y Pla-Sentis, I. 1992. Caracterización de los problemas de salinidad de suelos y aguas en cuatro zonas de la cuenca del lago de Maracaibo. Agronomía Tropical 42(1-2):85-96

Méndez N.; Ibarra P. y Merazo P. 2002. Germinación de semillas y desarrollo de plántulas de tres híbridos de maíz (Zea mays L.) bajo soluciones osmóticas II. Sulfato de sodio. VI 
Festival del Maíz y VI Jornada Científica Nacional del Maíz. http:// www.ceniap.gov.ve/pbd/Congresos/ jornada s\% $20 \mathrm{de} \% 20 \mathrm{maiz} /$ $6 \% 20 j o r n a d a s / c a r t e l e s / t e c n o s e m i l l a /$ jmendezsulfato.htm. [Accedido: 04 27 - 2007]

Moterle, L.; de Carvalho Lopes, P.; de Lucca e Braccini, A. y Scapim, C. 2006. Germinação de sementes e crescimento de plântulas de cultivares de milho-pipoca submetidas ao estresse hídrico e salino. Revista Brasileira de Sementes 28(3):169-176

Murillo-Amador, B. y Troyo-Diéguez, E. 2000. Effects of salinity on the germination and seedlings characteristics of cowpea [Vigna unguiculata (L.) Walp.]. Australian Journal of Experimental Agriculture 40(3):433-438

Musgrove, M.; Priestley, D. y Leopold, A. 1980. Methanol stress as a test of seed vigor. Crop Science 20(5):626-630

Porta C.; López-Acevedo R. y Roquero De L. 1999. Edafología. Mundi-Prensa, Madrid, p454; 657-705

Prisco, J.; Eneas Filho, J. y Gomes Filho, E. 1981. Effect of $\mathrm{NaCl}$ salinity on cotyledon starch mobilization during germination of Vigna unguiculata (L.) Walp seeds. Revista Brasileira de Botanica 4:63-71

Prisco, J. y O'leary, J. 1970. Osmotic and toxic effects of salinity on germination of Phaseolus vulgaris L. seeds. Turrialba 20:177-184

Rodrigues, L.; Fernandes, P.; Gheyi, H. y Viana, S. 2002. Germinação e formação de mudas de arroz irrigado sob estresse salino. Revista Brasileira de Engenharia Agrícola e Ambiental 6(3):397-403

Shonjani, S. 2002. Salt sensitivity of rice, maize, sugar beet, and cotton during germination and early vegetative growth. Dissertation im Fachbereich Agrarwissenschaften und Umweltsicherung. Institut für Pflanzenernährung der Justus-LiebigTesis Ph.D., Justus Liebig University, Giessen.

Da Silva, R.; Fernandes Lopes, N.; Munt de Moraes, D.; de Almeida Pereira, A. y Loureiro Duarte. G. 2007. Physiological quality of barley seeds submitted to saline stress. Revista Brasileira de Sementes 29(1):40-44.

Soltani, A.; Galeshi, S.; Zeinali, E. y Latifi, N. 2002. Germination, seed reserve utilization and seedling growth of chickpea as affected by salinity and seed size. Seed Science and Technology 30(1):51-60

Tobe, K.; Li, X. y Omasa, K. 2000. Seed germination and radicle growth of a halophyte, Kalidium caspicum (Chenopodiaceae). Annals of Botany 85:391-396

Wong R. 2002. Efecto de cinco potenciales osmóticos creados con $\mathrm{NaCl}$ y sacarosa comercial sobre la germinación de las semillas y desarrollo inicial de las plántulas de tres híbridos de maíz (Zea mays L.). Tesis Ingeniero Agrónomo, Universidad de Oriente, Monagas 